\title{
Impacto da Tecnologia Assistiva em indivíduos com Dislexia: uma Revisão Sistemática
}

\author{
Eliezer Araújo ${ }^{1}$, Raúl Teves², Roberto Machado ${ }^{3} \&$ Rui Rofino ${ }^{4}$
}

\begin{abstract}
Resumo
Esta revisão sistemática teve por objetivo verificar do impacto das diferentes formas de Tecnologia Assistiva existentes na reabilitação de indivíduos com Dislexia. Foram seguidas as orientações PRISMA para definição dos critérios de inclusão e exclusão dos artigos. Tais artigos foram selecionados através das seguintes bases de dados: B-on, PubMed, Scopus, Cochrane, Science.gov, CORE, ScienceDirect e Taylor \& Francis. O risco de enviesamento foi avaliado através do "Cochrane Risk of Bias Assessment Tool'. A combinação das amostras de todos os estudos incluiu um total de 344 participantes com dislexia, dos quais 323 participantes são crianças e adolescentes, e 21 são jovens adultos e adultos. Verificou-se que a maior parte dos estudos evidenciam um impacto positivo da TA ao nível da leitura. Considera-se que a presente revisão fornece um apanhado das investigações e softwares mais recentes no âmbito da reabilitação com TA e constitui um testemunho fundamentado que poderá consciencializar os profissionais em relação aos benefícios do uso da tecnologia.

Palavras-chave: Dislexia, Tecnologia Assistiva, Aplicações, Reabilitação.
\end{abstract}

Aveiro.

PhD by University of Coimbra. Dept. of Education and Psychology - University of

Department of Education and Psychology - University of Aveiro.

Department of Education and Psychology - University of Aveiro.

Department of Education and Psychology - University of Aveiro. 


\begin{abstract}
This systematic review aimed to verify the impact of different forms of Assisted Technology in the rehabilitation of individuals with dyslexia. PRISMA guidelines were followed to define inclusion and exclusion criteria for articles selected through the following databases: B-on, PubMed, Scopus, Cochrane, Science.gov, CORE, ScienceDirect and Taylor \& Francis. The risk of bias was assessed through the "Cochrane Risk of Bias Assessment Tool". The combination of samples from all studies included a total of 344 participants with dyslexia, of whom 323 were children and adolescents, and 21 were young adults and adults. It was found that most studies showed a positive impact of AT at the reading level. This review is considered to provide an overview of the latest research and software in the field of AT rehabilitation and to be an authoritative testimony that may raise awareness among professionals of the benefits of using the technology.
\end{abstract}

Keywords: Dyslexia, Assisted Technology, Applications, Rehabilitation.

\title{
Introdução
}

A Dislexia é uma Perturbação Específica da Aprendizagem (PEA) de origem neurológica que se caracteriza por dificuldades na leitura e que pode ter repercussão na escrita e na ortografia. Pode ser impactante em processos cognitivos como a memória, velocidade de processamento, bem como, a gestão de tempo, e pode estar relacionada com dificuldades visuais e/ou fonológicas (Cameirão, 2014).

Estima-se que a sua prevalência se situe entre os 7 e os $10 \%$ da população mundial (Hall, Smith, \& Wicaksono, 2016; Hulme \& Snowling, 2016), com maior incidência sobre o sexo masculino, e que haja uma concomitância amiúde com a Perturbação de Hiperatividade e Défice de Atenção e/ou com a Dispraxia (Hulme \& Snowling, 2016). Em Portugal, o estudo de Vale, Sucena e Viana (2011), propôs que a Dislexia afetaria cerca de $5.4 \%$ das crianças no primeiro ciclo de ensino básico, sendo a incidência mais frequente sobre o sexo masculino.

A perturbação pode ser classificada de acordo com a sua origem e seu tipo. No que toca à origem, é possível classificá-la como Dislexia do Desenvolvimento ou Dislexia Adquirida. Enquanto que a primeira se manifesta na infância e é caracterizada 
pela origem hereditária, a segunda está associada à lesão neurológica adquirida. No que respeita ao tipo, existem: a Dislexia Periférica, caracterizada pela dificuldade em analisar a forma da palavra; e a Dislexia Central, caracterizada pela dificuldade em pronunciar ou compreender as palavras (Coslett \& Turkeltaub, 2016). É importante que se entenda que Dislexia não é sinónimo de incapacidade intelectual, visto não haver comprometimento da inteligência.

Embora se trate de uma questão complexa, há evidências que comprovam a existência de uma etiologia genética que parece envolver os genes dominantes e recessivos (Cameirão, 2014). Para lá da hereditariedade, a ressonância magnética funcional tem se debruçado sobre o funcionamento neuronal das pessoas acometidas e tem vindo a demonstrar que a diferença mais comum entre um cérebro normal e um cérebro com dislexia, reside nas hipoativações que acontecem no giro frontal inferior e nas regiões posteriores do hemisfério esquerdo. Ainda dentro desta tónica referente à etiologia, é comum aludir-se a hipótese do défice fonológico. Défice que se pode manifestar de várias formas, nomeadamente através de: (i) défice na identificação e análise de fonemas (i.e., capacidade de identificar, segmentar e manipular os sons da fala); (ii) défice na memória verbal de curto prazo; e (iii) acesso lexical mais lento (e.g., tarefas de nomeação rápidas em série; Cameirão, 2014).

O diagnóstico da dislexia é considerado um "processo interativo". Numa primeira fase, sobreleva-se a necessidade de se identificarem dificuldades de leitura e ortografia por um período de seis ou mais meses - independentemente da existência de intervenções vigentes e focadas nessas dificuldades (American Psychiatric Association, 2014, pp.78). Numa segunda fase, é necessário que se assegure que a Dislexia não é explicada por outras perturbações, défices ou "falta de oportunidades (e.g., avaliação de instruções adequadas)". Por fim, numa terceira fase, é imperativo que se perceba se existem ou não défices cognitivos (De Jong \& Van Berger, 2017, pp.359).

A Reabilitação Neuropsicológica $(\mathrm{RN})$ na Dislexia trabalha os domínios da leitura, expressão oral (trabalhada ao longo da intervenção nos outros domínios), ortografia, expressão escrita, bem como a esfera emocional e afetiva. O diagnóstico precoce assume especial relevância, uma vez que pode dar azo a uma intervenção igualmente precoce e intensa. Tal necessidade surge porque o desenvolvimento biológico poderá dificultar a possibilidade de compensação das dificuldades advindas da condição, e porque quanto mais cedo houver intervenção, maior será a probabilidade de se evitar a experiência de insucesso académico. 
O trabalho sobre o âmbito da leitura deve ter em atenção os processos fonológicos (Medeiros, 2020). Assim, deve centrar-se não somente na compreensão, expressão oral, psicomotricidade e na esfera visual-auditiva, mas também no trabalho de regras e indicações ortográficas específicas (Sadasivan, 2013). As estratégias de intervenção poderão ser variadas, viz., exercícios de correspondência letra e som; segmentação fonológica (solicitar a audição de palavras posteriormente partidas em fonemas); leitura e soletração de palavras; instrução de palavras irregulares, onde o indivíduo deverá identificar palavras irregulares ditadas pelo instrutor no texto); mistura de fonemas; nomenclatura alfabética; e como não poderia deixar de ser, prática de leitura oral assistida.

No que toca ao domínio da ortografia, este influi negativamente sistemas como o fonológico, o visual (e.g., dificuldade em aprender sequências de letras), e o sistema de discurso (e.g., dificuldade em distinguir determinados sons). Aqui, a intervenção poderá usufruir de um conjunto de estratégias multissensoriais, como são exemplo as listas de palavras, estratégias visuais (e.g., o instrutor mostra a palavra; tapa-a; o indivíduo escreve a palavra; o instrutor faz correção), ou pistas de ortografia.

A expressão escrita, por seu turno, deve ser intervencionada através da prática frequente por parte do acometido. Este domínio envolve e pode comprometer diversos sistemas como a memória, a organização, o sequenciamento, a imaginação, a gramática/ sintaxe, o desenvolvimento de um fio condutor, ou a identificação de pontos chave. A intervenção deverá fomentar o gosto pela escrita através de simples estratégias como: fazer com que a pessoa pratique sobre temas que lhe interessem; e fazer com que examine o objetivo da redação, apresentando diferentes razões para a sua elaboração; entre outros. Grosso modo, é importante que se dê atenção à desmotivação da pessoa (advinda de fracassos repetidos) através da atribuição de sentido à prática. Outra estratégia importante é ajudar a internalizar uma estrutura com etapas para o desenvolvimento da escrita, uma vez que nas pessoas disléxicas é comum existir alguma inércia aquando da solicitação de uma tarefa deste tipo. Ainda no domínio da escrita, sabe-se que os indivíduos com Dislexia têm uma consciência metacognitiva (i.e., conhecimento ou a consciência e controlo dos processos cognitivos) pouco desenvolvida por conta das dificuldades fonológicas. Nas estratégias metacognitivas para a escrita, o objetivo é ajudar ao indivíduo a praticar através de um processo que deverá incluir: (i) a externalização em voz alta do pensamento enquanto escreve - à semelhança do que se faz nos treinos de auto-instrução; (ii) o exame de forma crítica e 
revisão das decisões de escrita, por exemplo, questionando "porque é que escrevi isto?"; e (iii) a antecipação de eventuais dificuldades para o leitor. Esta técnica promove a autonomia e a reflexão. A isto também se acrescenta as dificuldades ao nível da resolução de problemas - pessoas com Dislexia tendem a utilizar muitos caminhos para chegar a um mesmo fim. Aqui, o Treino de Resolução de Problemas poderá ser uma mais-valia (Reid, 2009).

Nota para Christ e colegas (2013), que defendem que o estabelecimento de objetivos é algo muito importante para o trabalho junto das pessoas com Dislexia (e.g., na elaboração de um currículo base voltado para o auxílio na leitura oral). A determinação de objetivos específicos, realistas e alcançáveis poderá desempenhar um papel muito importante porque poderá evitar inúmeras experiências indesejáveis como, por exemplo, as de frustração (abordadas abaixo).

Livingston e colaboradores (2018) sugerem uma associação entre problemas emocionais e indivíduos com Dislexia, muito em virtude de uma autoavaliação deturpada que poderá ter origem (e relação) em diversas consequências primárias (e.g., desempenho académico/trabalho; estigma; etc.) e secundárias (e.g., desmotivação, baixa autoestima, bem-estar emocional deficiente, conduta desadaptativa, etc.) resultantes da sintomatologia. Hendren e colegas (2018), sugerem que pode haver comprometimento do processamento emocional e que é comum encontrar altos níveis de stress e ansiedade, bem como de maior suscetibilidade à depressão nos disléxicos. Para Livingston e colegas (2018), os sentimentos de ansiedade e tristeza poderão estar associados às dificuldades de aprendizagem e produzem um impacto negativo no bemestar geral e na qualidade de vida. Tal desajustamento emocional, de acordo com os autores, poderá propulsionar o desinteresse e atitudes negativas face à leitura; a frustração face ao desempenho escolar; o abandono escolar; a auto-etiquetagem; e o desenvolvimento de perturbações psiquiátricas. Por este motivo, apontam que o ajustamento emocional pode ser um importante fator de avaliação das intervenções realizadas em indivíduos com dislexia. A intervenção de cariz psicoterapêutica deverá primar pelo estabelecimento de uma boa aliança terapêutica com cliente, pais e professores, e pelo envolvimento dos pais na leitura (no caso das crianças); pela utilização de sistemas de recompensa; pelo estabelecimento de rotinas (Jongsma, Peterson, \& Mcinnis, 2014); pela promoção do autoconceito e autorregulação (Graham \& Berman, 2012); bem como pela abordagem multidisciplinar, onde a inclusão da terapia da fala é muito importante (Medeiros, 2020). 
Muita da intervenção realizada no âmbito da reabilitação segue métodos mais tradicionais com recurso, por exemplo, a lápis e papel (Reid, 2009). Todavia, mais recentemente, investigadores e interventores têm vindo a usufruir de métodos de trabalho que incluem a tecnologia (Galuschka et al., 2020). A tecnologia utilizada no âmbito da reabilitação dá pelo nome de Tecnologia Assistiva (TA). A TA é nada menos do que uma área do conhecimento que tem como propósito a promoção ou o fornecimento de habilidades aos indivíduos comprometidos a nível funcional (Federici \& Scherer, 2018). Segundo Edyburn (2015), esta tem o potencial de fazer com que tais indivíduos sejam capazes de viver, aprender e trabalhar de forma autónoma, através de tecnologias que reduzem, eliminam ou minimizam o impacto das dificuldades ou incapacidades existentes. De acordo com Federici e Scherer (2018), a TA tem um papel fundamental na integração social das pessoas com incapacidade física, sensorial, comunicativa ou cognitiva. Os videojogos, por exemplo, têm-se mostrado uma forma de reabilitação atraente para as crianças, havendo inclusive estudos que demonstram o seu impacto positivo nas habilidades de leitura (e.g., Ronimus et al., 2019; Svenson et al., 2018). A intervenção na Dislexia pode beneficiar com o uso de TA, uma vez que permite não só moldar a curva da aprendizagem, como poderá também ser uma forma divertida de integração no processo de aprender. $\mathrm{O}$ facto de fomentar o compromisso e envolvimento a longo-prazo com as tarefas (e.g., repetição dos exercícios), e o facto de que diversas aplicações ou softwares podem ser utilizados em diferentes dispositivos, também é algo que pode facilitar o processo de reabilitação. A isto, acrescente-se ainda que a TA pode ser uma ferramenta de útil auxílio no que respeita ao estabelecimento de diagnóstico de forma mais rápida e eficaz (Rauschenberger, Baeza-Yates, \& Rello, 2019).

O objetivo da presente revisão sistemática prende-se com a verificação do impacto das diferentes formas de TA (e.g., aplicações para smartphones, ferramentas diversas para computadores, jogos, entre outros) na reabilitação de indivíduos com Dislexia. 


\section{Metodologia}

\section{Critérios de elegibilidade}

Como critérios de inclusão da presente revisão sistemática, elaborados segundo o PRISMA, definiram-se: (i) estudos que incluíssem indivíduos diagnosticados com dislexia; (ii) estudos que mostrassem o impacto do uso da TA em indivíduos com dislexia; (iii) estudos quasi-experimentais e/ou experimentais; (iv) estudos publicados em revistas académicas; (v) estudos de língua espanhola, inglesa ou portuguesa; e, por fim, (vi) estudos datados com menos de 5 anos. Como critérios de exclusão foram: (i) revisões sistemáticas; (ii) estudos que não fossem randomizados ou controlados; (iii) estudos sem foco na TA; e (iv) estudos cujos grupos de controlo fossem submetidos a intervenção com TA.

\section{Tabela 1}

Modelo PICO de revisão sistemática

\begin{tabular}{cccc}
\hline População & Intervenção & Comparador & Resultados \\
\hline $\begin{array}{c}\text { Indivíduos } \\
\text { diagnosticados com } \\
\text { dislexia }\end{array}$ & Tecnologia Assistiva & $\begin{array}{c}\text { Utilização vs. Não } \\
\text { utilização de TA }\end{array}$ & $\begin{array}{c}\text { Impacto da } \\
\text { intervenção }\end{array}$ \\
\hline
\end{tabular}

\section{Fontes de informação}

Os estudos foram selecionados por quatro investigadores de forma independente através das bases de dados integrativas: B-on, PubMed, Scopus, Cochrane, Science.gov, CORE. Também foram utilizadas bases de dados editoriais, viz., ScienceDirect e Taylor \& Francis. Os artigos selecionados datam um intervalo temporal entre 2015 e 2019, e têm o inglês como idioma principal. A pesquisa foi efetuada de 2 de março a 24 de abril de 2020. 


\section{Estratégia de pesquisa}

Os estudos foram selecionados através da utilização das seguintes palavraschave: ("dyslexia" AND "technology" AND "rehabilitation"); ("dyslexia" AND "assistive technology"); ("dyslexia" AND "technology"); ("reading and writing disabilities" AND "assistive technology" AND “intervention"); ("assistive technology" AND "reading intervention" AND "dyslexia"); ("dyslexia" AND "technology" AND ("videogames" OR “application")); (“assistive technology" AND "reading intervention" AND (“videogames" OR “application”)).

\section{Seleção dos estudos}

\section{Controlo de dados}

Um dos autores sumarizou e organizou os resultados das pesquisas no Excel assinalando: a base de dados; o número de artigos selecionados pelo título, abstract e conteúdo; o número de artigos duplicados; e a seleção final dos artigos. Neste documento também foram assinaladas as situações de exclusão consequentes da análise dos critérios de elegibilidade.

\section{Processo de recolha de dados}

Para obtenção de dados, cada investigador realizou a pesquisa de forma independente, selecionando um conjunto de artigos pelo título - posteriormente reduzido por conta da leitura do abstract. De seguida, cada membro da equipa procedeu à leitura na íntegra dos artigos por si selecionados de forma a decidir quais os que seriam passíveis de partilha intergrupal. Posteriormente, todos os investigadores procederam à leitura dos 23 artigos finais para seleção da informação considerada relevante. Por fim, os conteúdos destacados foram cruzados, chegando-se a um consenso acerca das informações e dos artigos que se mostraram efetivamente pertinentes (cf. Figura 1). Denote-se que cada autor procedeu ao registo dos conteúdos extraídos dos estudos num documento Word partilhado online. Tal registo continha informação referente à identificação do estudo, ao método, aos objetivos, aos participantes e suas 
características, ao tipo de intervenção, às medidas de resultados, aos resultados, às limitações e às implicações futuras.

\section{Lista de dados}

Os principais achados da presente revisão sistemática constam na tabela $2^{5}$. No geral, os estudos procuraram avaliar a eficácia de diferentes tipos de TA nas dificuldades de leitura em indivíduos com dislexia, sendo que um também trabalhou as questões do autoconceito, autoimagem e saúde mental. A combinação das amostras de todos os estudos inclui um total de 344 participantes com dislexia, dos quais 323 participantes são crianças e adolescentes, e 21 são jovens adultos e adultos. O estudo de Caute e colaboradores (2018) trabalhou especificamente com adultos com dislexia adquirida após acidente vascular cerebral (AVC) $(n=21)$.

\section{Risco de enviesamento em cada estudo}

Dos quatro estudos selecionados, um é quasi-experimental e três experimentais (RCT). A determinação destas categorias teve como base as checklists do manual "The Joanna Briggs Institute Reviewer's" (Tufanaru et al., 2020; cf. Anexo A e B). No que toca ao risco de enviesamento em cada estudo, a deliberação da presente revisão teve como base os princípios orientadores da "The Cochrane Collaboration's tool for assessing risk of bias in randomised trials". Esta ferramenta possui sete itens passíveis de classificação que poderá determinar se o risco de enviesamento é "baixo", "alto" ou se a informação fornecida é inconclusiva, ou seja, "não é claro" (cf. Figura 2). Denotese que esta análise incide somente sobre os estudos experimentais (Higgins et al., 2011).

Para leitura detalhada de cada estudo selecionado, consultar anexo C 


\section{Figura 1}

Diagrama de fluxo do processo de identificação dos artigos para a revisão sistemática

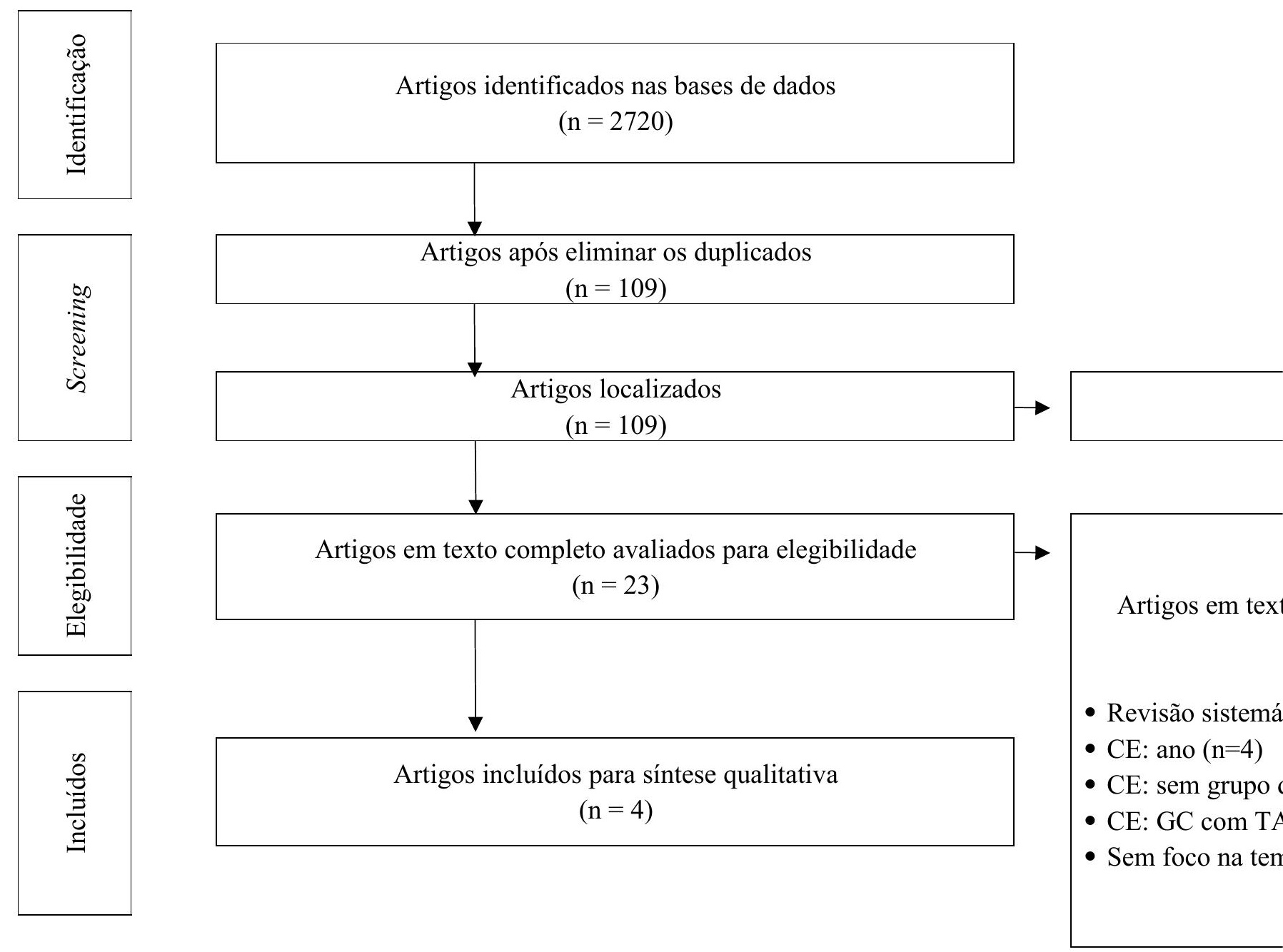

Figura 1. Diagrama de fluxo do processo de identificação dos artigos para a revisão sistemática com base na excluído por não respeitar os critérios de elegibilidade. Adaptado de "The PRISMA statement for reporting system that evaluate healthcare interventions: explanation and elaboration" de A. Liberati, D. Altman, J. Tetzlaff, C. Mur P. Devereaux, J. Kleijnen, \& D. Moher, 2009, BMJ, 339(b2700)

\section{Tabela 2}

Lista de dados

\begin{tabular}{lllll}
\hline $\begin{array}{c}\text { Identificaçã } \\
\text { o }\end{array}$ & \multicolumn{1}{c}{ Participantes } & \multicolumn{1}{c}{ Design } & \multicolumn{1}{c}{ Objetivos } \\
\hline $\begin{array}{l}\text { Caute } \\
\text { et al. }(2018)\end{array}$ & $\begin{array}{l}21^{\text {ac }} \\
(\mathrm{n} / \mathrm{e})\end{array}$ & $\begin{array}{l}\text { Quasi- } \\
\text { experimental }\end{array}$ & $\begin{array}{l}\text { Avaliar o efeito da TA na leitura na dislexia adquirida } \\
\text { após AVC }\end{array}$ & $C$ \\
Lindeblad & $137^{\text {bd }}$ & Experimental & Avaliar o impacto da TA na autoimagem, autoconceito, & $K$
\end{tabular}


et al. (2019) $\quad(\mathrm{n} / \mathrm{c})$

Ronimus 37

$37^{\mathrm{b}}$

GT 19; GC

20)

Svensson $\quad 149^{\mathrm{b}}$

et al. (2019) (GT 71; GC 78) saúde mental e dificuldade de leitura

Experimental Avaliar a eficácia de um videojogo como ferramenta de apoio para a leitura.

Experimental

Explorar os efeitos da TA em estudantes com dificuldades severas na leitura

Legenda: ${ }^{a}$ jovens adultos/adultos; ${ }^{\mathrm{b}}$ crianças/adolescentes; ${ }^{\mathrm{c}}$ Não especifica; ${ }^{\mathrm{d}}$ Não é claro 


\section{Figura 2}

Análise do risco de enviesamento

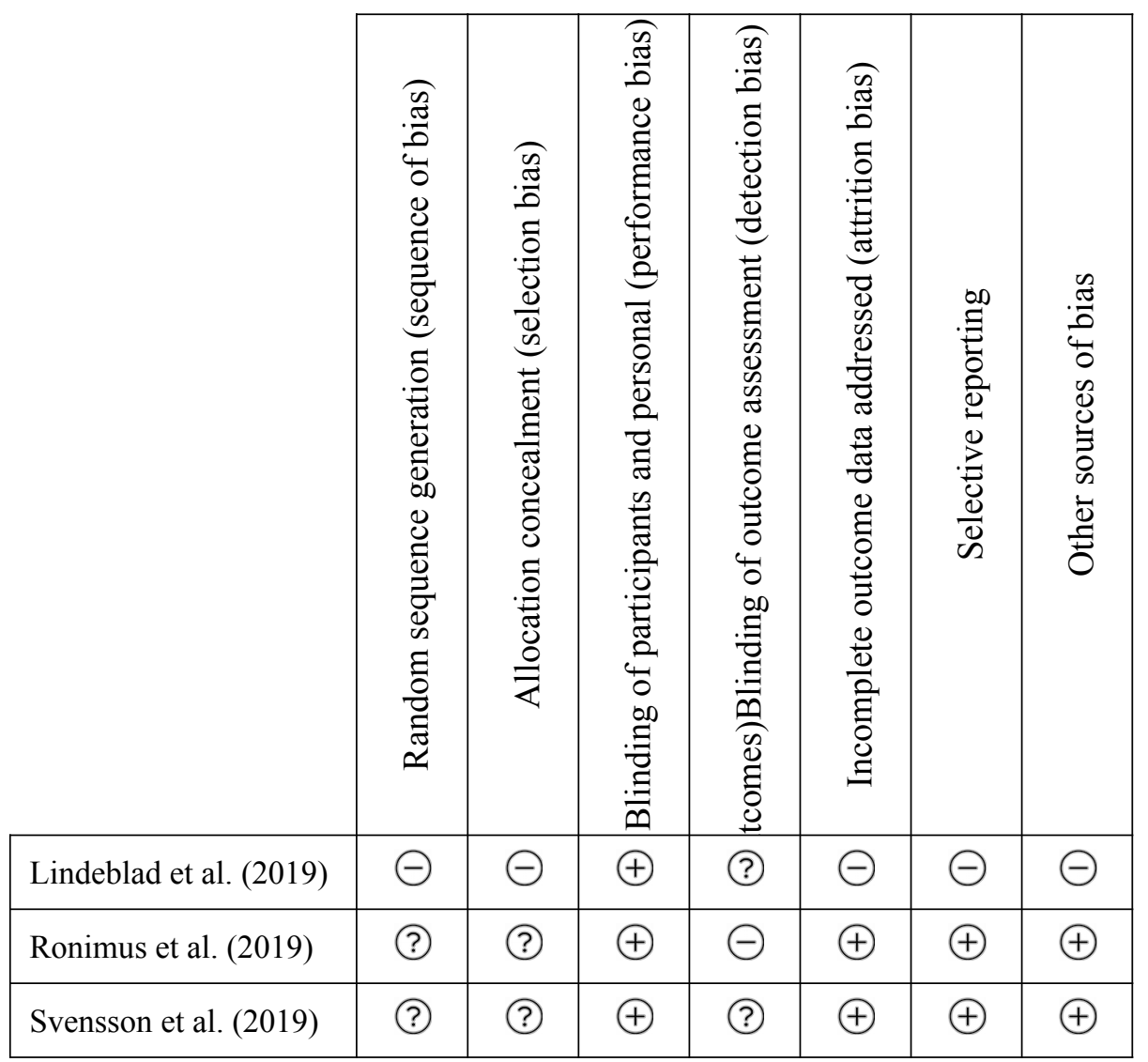

Figura 2. Análise do risco de viés. $\oplus$ Baixo risco de viés; $\odot$ Alto risco de viés; ( ₹ Não é claro. Adaptado de "The Cochrane Collaboration's tool for assessing risk of bias in randomised trials and meta-analyses of studies that evaluate healthcare interventions: explanation and elaboration" de P. Higgins, D. Altman, P. Gøtzsche, P. Jüni, D. Moher, A. Oxman, J. Savović, K. Schulz, L. Weeks, J. Sterne, Cochran Bias Methods Group, \& Cochrane Statistical Methods Group, 2011, BMJ, 343(d5928), p. 9.

\section{Resultados}

No estudo de Caute e colaboradores (2018), os resultados sugerem que tanto a compreensão da leitura, a autoconfiança, como as emoções associadas à leitura, melhoraram após a intervenção com tecnologia assistiva. As avaliações realizadas no pós-teste e follow-up, mostram que os participantes pertencentes ao GT melhoraram significativamente a sua capacidade de leitura - fenómeno não verificado nos participantes pertencentes ao GC. 
Por sua vez, os resultados do estudo de Lindeblad e colegas (2019) não só evidenciam a inexistência de qualquer impacto das intervenções com TA na autoestima e autoconceito, como também acrescentam que as pontuações no teste de capacidade de leitura não se correlacionam com as notas obtidas nos testes de autoconceito. De referir que os resultados não são claros no que diz respeito ao impacto sobre as dificuldades de leitura.

No estudo de Ronimus (2019), os resultados mostraram que a exposição de seis semanas à intervenção com o GraphoLearn melhorou a capacidade de leitura das crianças do GT. Mesmo em situações onde o desenvolvimento das habilidades de leitura e de ortografia do GT tenha sido significativamente mais lento (viz., follow-up), os participantes foram capazes de alcançar o nível pretendido de desempenho. Os resultados mostraram ainda que o tempo de treino (com a aplicação) não é preditor da manutenção da aprendizagem per se.

No que concerne ao estudo de Svensson e colaboradores (2019), os resultados sugerem melhorias para o GT no que toca à capacidade de leitura durante o período de intervenção - em comparação com o GC. Todavia, os autores não encontraram diferenças entre os grupos e as idades aquando da avaliação do pós-teste e do follow-up. Denote-se que o GC recebeu intervenção tradicional. Os resultados também sugerem que o uso de TA permite remediar as dificuldades no processo de codificação em crianças que até ao $3^{\circ}$ ano não tenham conseguido alcançar o nível desejado de leitura para a sua idade. Por fim, mostram a existência daquilo que parece ser um incremento na autonomia e motivação escolar dos participantes após o término das intervenções.

\section{Discussão}

Todos os estudos, à exceção de Lindeblad e colaboradores (2019), evidenciaram aquilo que parece ser um impacto positivo da TA ao nível da leitura. $\mathrm{O}$ artigo de Ronimus e colegas (2019) acrescenta um impacto positivo também ao nível da ortografia.

No geral, a TA parece ser eficiente para a população quer em contexto de sala de aula, quer em contexto doméstico. Destaque para este último, uma vez que há inclusão manifesta do auxílio dos pais. Tal facto poderá assumir um carácter tanto de limitação como de mais valia. Por um lado, limitação, porque os mesmos poderão impactar negativamente o treino das crianças se não forem devidamente instruídos ou se 
não deterem habilidades adequadas para o uso da tecnologia empregue na intervenção. Por outro lado, uma mais valia, porque poderão desempenhar um papel muito relevante ao nível do suporte social - assumido como um dos aspetos fortes para o sucesso da reabilitação (Wade \& Hung, 2015).

Os dados apurados do estudo de Svensson e colaboradores (2019) mostraram ainda que a intervenção com crianças através de videojogos parece promover o aumento da motivação e da autonomia. Segundo Martí-Parreño e colaboradores (2018), estes constructos correlacionam-se fortemente com altos níveis de autoeficácia que, por sua vez, de acordo com Bandura (2010), se traduzem num incremento da tolerância à frustração. Denote-se que este incremento culmina naturalmente em benesse para os indivíduos acometidos com dislexia, uma vez que estes experienciam amiúde intensos níveis de intolerância à frustração.

Grosso modo, os resultados encontrados na presente revisão sistemática vão ao encontro aos resultados da meta-análise elaborada por Sim e Walker (2014), que inclui investigações realizadas entre 2006 e 2010 . Embora os softwares utilizados nas mesmas sejam diferentes, os resultados da pesquisa sugerem de igual modo o impacto positivo da utilização de TA em indivíduos com dislexia. O mesmo se diga da revisão de Rauschenberger e colaboradores (2019), que incluí estudos realizados desde 2005.

\section{Limitações}

Como primeira limitação assinalável dos estudos no geral, destaque para o atrito encontrado, uma vez que a maioria apresenta drop-out durante a intervenção e aquando o follow-up - os próprios autores recomendam a replicação das suas investigações.

Uma outra limitação encontrada prende-se com a inexistência de tónica dada à questão do sexo. Tendo em conta que a literatura evidencia que existem diferenças na prevalência da dislexia no que toca ao sexo (Hulme \& Snowling, 2016), e que estudos apontam para uma diferença entre sexos no que toca ao jogo de videojogos (Choi et al., 2020; Smohai et al., 2017), os artigos selecionados nesta revisão poderão ter negligenciado um tópico que, per se, poderá constituir um preditor de adesão à reabilitação com TA (Kaye, Yeager, \& Reed, 2008).

Assume-se ainda o facto de, por vezes, não haver clareza sobre alguns instrumentos utilizados. Ou seja, podem ter sido utilizados instrumentos distintos com parâmetros de interpretação dos resultados diferentes para medir o mesmo construto 
(e.g., avaliação da capacidade de leitura). De referir também a não exposição e a falta de clareza em relação ao número de participantes alocados nos GC e GT [viz., Caute e colaboradores (2018); e Lindeblad e colaboradores (2019)].

Por fim, no que toca à elaboração da presente revisão, considera-se que o número total da amostra é baixo $(\mathrm{n}=344)$ - o que poderá colocar em causa a direção dos resultados obtidos. A isso acrescente-se, embora se tenham apurado resultados maioritariamente baixos ou inconclusivos relativos ao risco de enviesamento (cf. Figura 2), o número reduzido de artigos constituintes da seleção final. Não obstante, é importante salientar que esse número se deve ao rigor imposto e respeitado ao longo de todo o trabalho. A seleção obedece na íntegra à lista de critérios definidos a priori do início da pesquisa e alguns artigos foram excluídos aquando da fase de elegibilidade por não terem grupo de controlo, ou por o terem submetido à intervenção com TA. Nota ainda para a possibilidade de viés de língua, uma vez que não foram equacionados estudos cuja linguagem estivesse fora do alcance de compreensão dos investigadores; e para o viés em relação ao intervalo temporal definido, i.e., a decisão de inclusão apenas de estudos realizados nos últimos cinco anos, poderá ter proporcionado a exclusão de investigações importantes, pese embora, mais antigas.

Assim sendo, recomendam-se futuras revisões com novos critérios, que incluam mais estudos com a mesma linha metodológica dos artigos eleitos.

\section{Implicações futuras}

Até ao momento, as aplicações utilizadas na TA focam-se nos problemas de escrita e leitura, pelo que ainda pouco se sabe acerca do seu impacto noutros domínios (e.g., social). Nesta linha de raciocínio a presente revisão poderá servir de incentivo para futuras investigações que trabalhem nesse sentido.

Em jeito de conclusão, considera-se a proficuidade da presente revisão, não só porque fornece um apanhado das investigações e softwares utilizados mais recentes no âmbito da reabilitação com $\mathrm{TA}$, mas também porque constitui um testemunho fundamentado que poderá consciencializar os profissionais acerca dos benefícios do uso da tecnologia. 


\section{Referências}

American Psychiatric Association. (2014). DSM-5: Manual de diagnóstico e estatística de perturbações mentais ( $\left.5^{\mathrm{a}} \mathrm{ed}\right)$. Cliempsi Editores.

Aravena, S., Tijms, J., Snellings, P., \& Van der Molen, M. (2016). Predicting responsiveness to intervention in dyslexia using dynamic assessment. Learning and Individual Differences, 49, 209-215. https://doi.org/10.1016/j.lindif.2016.06.024

Bandura, A. (2010). Self-efficacy. In B. Weiner \& W. Craighead, The Corsini Encycolpedia of Psychology $\left(4^{\text {th }}\right.$ ed). John Wiley \& Sons, Inc.

Cameirão, M., L. (2014). Dificuldades específicas de aprendizagem: Dislexia e Discalculia. In P. Monteiro (Coord.), Psicologia e Psiquiatria da Infância e Adolescência (1ª ed., pp. 101-15). Lidel.

Carawan, L. W., Nalavany, B. A., \& Jenkins, C. (2016). Emotional experience with dyslexia and self-esteem: the protective role of perceived family support in late adulthood. Aging \& Mental Health, 20(3), 284-94. https://doi.org/10.1080/13607863.2015.1008984

Caute, A., Cruice, M., Marshall, J., Monnelly, K., Wilson, S., \& Woolf, C. (2018). Assistive technology approaches to reading therapy for people with acquired dyslexia. Aphasiology, 32(1), 40-2. https://doi.org/10.1080/02687038.2018.1489119

Choi, E., Shin, S.-H., Ryu, J.-K., Jung, K.-I., Kim, S.-Y., \& Park, M.-H. (2020). Commercial video games and cognitive functions: video game genres and modulating factors of cognitive enhancement. Behavioral and Brain Functions, 16(2), 1-14. https://doi.org/10.1186/s12993-020-0165-Z

Christ, T. J., Zopluoglu, C., Monaghen, B., \& Van Norman, E. R. (2013). Curriculumbased measurement of oral reading (CBM-R) progress monitoring: Multi-study evaluation of schedule, duration, and dataset quality on progress monitoring outcomes. Journal of School Psychology, 51, 19-57. https://doi.org/10.1016/j.jsp.2012.11.001 
Coslett, H., \& Turkeltaub, P. (2016). Acquired dyslexia. In G. Hickok \& S. Small (eds.), Neurobiology of Language (pp.791-803). Academic Press.

De Jong, P., \& van Bergen, E. (2017). Issues in diagnosing dyslexia. In E. Segers \& P. Van den Broek (Eds.), Developmental perspectives in written language and literacy (pp. 349-361). John Benjamins Publishing Company.

Edyburn, D. L. (2015). Expanding the use of assistive technology while mindful of the need to understand efficacy. In D. Edyburn (Ed.), Efficacy of assistive technology interventions (pp. 1-12). Emerald Group Publishing Limited.

Federici, S., \& Scherer, M. J. (2018). The Assistive Technology Assessment Process Model and Basic Definitions. In S. Federici, \& M. J. Scherer (Eds.), Assistive Technology Assessment Handbook ( $2^{\text {nd }}$ ed., pp. 1-9). Taylor \& Francis Group.

Galuschka, K., Görgen, R., Kalmar, J., Haberstroh, S., Schmalz, X., \& Schulte-Körne, G. (2020). Effectiveness of spelling interventions for learners with dyslexia: A meta-analysis and systematic review. Educational Psychologist, 55(1), 1-20. DOI:10.1080/00461520.2019.1659794

Graham, L., \& Berman, J. (2012). Self-regulation and learning disabilities. Social Education Perspectives, 21(2), 42-52.

Gribben, M. (2012). The Study Skills Toolkit for Students with Dyslexia. SAGE Publications.

Hall, C., Smith, P., \& Wicaksono, R. (2016). Mapping applied linguistics: a guide for students and practitioners ( $\left.2^{\text {nd }} \mathrm{ed}\right)$. Routledge.

Hargreaves, S., \& Crabb, J. (2016). Study Skills for Students with Dyslexia: Support for Specific Learning Differences ( $3^{\text {rd }}$ ed). SAGE Publications.

Hendren, R. L., Haft, S. L., Black, J. M., White, N. C., \& Hoeft, F. (2018). Recognizing psychiatric comorbidity with reading disorders. Frontiers in Psychiatry, 9(101), 1-10. https://doi.org/10.3389/fpsyt.2018.00101

Higgins, J. P., Altman, D., Gøtzsche, P., Jüni, P., Moher, D., Oxman, A., Savović, J., Schulz, K., Weeks, L., Sterne, J., Cochrane Bias Methods Group, \& Cochrane Statistical Methods Group. (2011). The Cochrane Collaboration's tool for 
assessing risk of bias in randomised trials. BMJ, 343(d5928), 1-9. https://doi.org/10.1136/bmj.d5928

Hulme, C., \& Snowling, M. J. (2016). Reading disorders and dyslexia. Current Opinion in Pediatrics, 28(6), 731-5. http://dx.doi.org/10.1097/MOP.0000000000000411

Jongsma, A. E., Peterson, L. M., McInnis, W. P. (2014). PracticePlanners ${ }^{\circledR}-$ Child Psychotherapy Homework Planner $\left(5^{\text {th }}\right.$ ed). Wiley.

Kaye, H., Yeager, P., \& Reed, M. (2008). Disparities in usage of assistive technology among people with disabilities. Assistive Technology, 4, 194-203. https://doi.org/10.1080/10400435.2008.10131946

Liberati, A., Altman, D., Tetzlaff, J., Murlow, C., Gøtzsche, O., Ioannidis, J., Clarke, M., Devereaux, P., Kleijnen, J., \& Moher, D. (2009). The PRISMA statement for reporting systematic reviews and meta-analyses of studies that evaluate healthcare interventions: explanation and elaboration. BMJ, 339(b2700). https:// doi.org/10.1371/journal.pmed.1000100

Lindeblad, E., Nilsson, S., Gustafson, S., \& Svensson, I. (2019). Self-concepts and psychological health in children and adolescents with reading difficulties and the impact of assistive technology to compensate and facilitate reading ability, Cogent Psychology, 6(1), 1-18. https://doi.org/10.1080/23311908.2019.1647601

Livingston, E. M., Siegel, L. S., \& Ribary, U. (2018). Developmental dyslexia: emotional impact and consequences. Australian Journal of Learning Difficulties, 23(2), 107-35. https://doi.org/10.1080/19404158.2018.1479975

Martí-Parreño, J., Galbis-Cordová, A., \& Miquel-Romero, M. (2018). Students' attitude towards the use of educational video games to develop competencies. Computers in Human Behavior, 81, 366-77. https://doi.org/10.1016/j.chb.2017.12.017

Medeiros, T. (2020). O neuropsicólogo e as perturbações da aprendizagem específicas. In R. Bonança, T. Medeiros, \& T. Botelho, Referencial de boas práticas: As perturbações da aprendizagem específicas e os princípios da educação inclusiva (1st ed., pp. 40-2). Disbedo. 
Rauschenberger, M., Baeza-Yates, R., \& Rello, L. (2019). Technologies for Dyslexia. In Y. Yesilada. \& S. Harper (Eds.), Web Accessibility (pp. 603-27). HumanComputer Interaction Series.

Reid, G. (2009). Dyslexia: a practitioner's handbook (4th ed). John Wiley \& Sons Ltd.

Ronimus, M., Eklund, K., Pesu, L., \& Lyytinen, H. (2019). Supporting struggling readers with digital game-based learning. Educational Technology Research and Development, 67, 639-63. https://doi.org/10.1007/s11423-019-09658-3

Sadasivan, A. (2013). Neuropsychological Intervention for Specific Learning Disorder: An Innovative Approach. In: J. Rajeswaran (Ed.), Neuropsychological Rehabilitation Principles and Applications (pp. 155-76). Elsevier.

Schmitt, A. J., McCallum, E., Hennessey, J., Lovelace, T., Hawkins, R. O. (2012). Use of reading pen assistive technology to accommodate post-secondary students with reading disabilities. Assistive Technology, 24(4), 229-39. http://dx.doi.org/10.1080/10400435.2012.659956

Semrud-Clikeman, M. \& Fine, J. (2008). Brief and comprehensive neuropsychological assessment of children and adults with learning difficulties and disabilities. In: R. D’Amato \& L. Hartlage (Eds.), Essentials of neuropsychological assessment: treatment planning for rehabilitation (2nd ed., pp. 245-92). Springer Publishing Company.

Sim, T., \& Walker, Z. (2014). A meta-analysis of technology-based interventions on the phonological skills of children with dyslexia. Asia Pacific Journal of Developmental Differences, 190-201. https://dx.doi.org/10.3850/S2345734114000043

Smohai, M., Urbán, R., Griffiths, M., Király, O., Mirnics, Z., Vargha, A., \& Demetrovics, Z. (2017). Online and offline video game use in adolescents: measurement invariance and problem severity. American Journal of Drug and Alcohol Abuse, 43(1), 111-6. https://doi.org/10.1080/00952990.2016.1240798

Svensson, I., Nordström, T., Lindeblad, E., Gustafson, S., Björn, M., Sand, C., Almgren/Bäck, G., \& Nilsson, S. (2019). Effects of assistive technology for students with reading and writing disabilities. Disability and Rehabilitation: Assistive Technology, 1-13. https://doi.org/10.1080/17483107.2019.1646821 
Tufanaru C., Munn Z., Aromataris E., Campbell J., \& Hopp L. (2020). Chapter 3: Systematic reviews of effectiveness. In E. Aromataris \& Z. Munn (Eds.), The Joanna Briggs Institute Reviewer's Manual (pp. 72-134). The Joanna Briggs Institute. https://reviewersmanual.joannabriggs.org/

Vale, A., Sucena., A., \& Viana, F. (2011). Prevalência da Dislexia entre Crianças do 1. ${ }^{\circ}$ Ciclo do Ensino Básico falantes do Português Europeu. Revista Lusófona de Educação, 18, 45-56. http://hdl.handle.net/1822/17195

Wade, S., \& Hung, A. (2015). Online Family Problem Solving for Adolescent Traumatic Brain Injury. In J. Reed, K. Byard \& H. Fine (Eds.), Neuropsychological Rehabilitation of Childhood Brain Injury (pp. 43-60). Plagrave MacMillan.

Nota: as referências estão de acordo com as normas da Publication Manual of the American Psychological Association, Seventh Edition (APA 7; 2020) 